\title{
Looking for cosmic ray data? The ASI Cosmic Ray Database
}

\author{
Valeria Di Felice ${ }^{1,2}$, Cecilia Pizzolotto ${ }^{2,3}$, Domenico D’Urso*2,4,5, Simonetta Dari ${ }^{6}$, \\ Daniele Navarra ${ }^{6}$, Roberto Primavera ${ }^{7}$, Bruna Bertucci ${ }^{2,3,8}$
}

${ }^{1}$ INFN, Sezione di Roma Tor Vergata, I-00133 Rome, Italy

${ }^{2}$ Agenzia Spaziale Italiana (ASI), Space Science Data Center, I-00044 Frascati, Italy

${ }^{3}$ INFN Sezione di Perugia, I-06100 Perugia, Italy

4 Universita' di Sassari, I-07100 Sassari, Italy

${ }^{5}$ INFN-LNS, I-95123 Catania, Italy

${ }^{6}$ Serco SpA, I-00044 Frascati, Rome, Italy

7 Telespazio a Leonardo/Thales Company, I-00156 Rome, Italy

${ }^{8}$ Universita' di Perugia, I-06100 Perugia, Italy

E-mail: valeria.difeliceeroma2.infn.it

A new Cosmic Ray DataBase (CRDB) is under development at the Space Science Data Center, a facility of the Italian Space Agency (ASI) that provides support to several space missions and acts as a multi-mission science operation, data processing and data archiving center. To ensure a more extensive utilisation of space data from existing, archived and future missions, on-line access to archival data, analysis software, calibration files and documentation are provided by a MultiMission Interactive Archive (MMIA) of the Space Science Data Center. Space data from different missions and in different research fields (astro-physics, astro-particle, cosmology, planetology) are connected in the MMIA in order to stimulate their cross-analysis and comparison. In this context, the CRDB aims to provide the scientific community with tools for an easy and efficient access to published data from missions dedicated to charged Cosmic Rays measurements. Originally developed to support the retrieval of PAMELA and AMS-02 results, it is now expanding to include more data sets from other experiments and evolving to provide new tools for data retrieval, visualisation and download. As an example, measurements of differential energy fluxes and flux ratios for the different species are available as a function of various parameters (e.g. energy, rigidity, time, magnetic parameters), for particles of galactic and solar origin, as well as particles trapped in the Earth magnetosphere. Data are ready for retrieval, visualisation and download. In this contribution, we will introduce the main features of the CRDB, accessible online at http://tools.asdc.asi.it/CosmicRays/, and the perspectives for its future development.

35th International Cosmic Ray Conference - ICRC2017

10-20 July, 2017

Bexco, Busan, Korea

${ }^{*}$ Speaker. 


\section{Introduction}

For decades, since the beginning of the space age, instruments devoted to study the charged component of cosmic rays have been collecting data, exploring orders of magnitude in energy and intensity, in order to provide insights on the details of cosmic ray spectra. Scientific exploitation of an ever increasing volume of data greatly benefits from methods for data access and visualization favouring their easy retrieval, combination and comparison.

In this context, the Space Science Data Center (SSDC) acts as a multi-mission data processing, data archiving and science operation center providing support to several space missions. In particular, the center hosts the Cosmic Ray DataBase (CRDB)[1], conceived as a tool to provide an easy and efficient access to published data from missions dedicated to charged cosmic ray measurements. The CRDB has been originally developed to support the retrieval of PAMELA and AMS-02 published data.

The PAMELA mission was conceived to search for primordial antimatter and indirect dark matter signals [2]. In order to do so the instrument, a magnetic spectrometer, was optimized for the detection of the antiparticle component of cosmic rays in the energy range between a few tens of $\mathrm{MeV}$ up to $\sim \mathrm{TeV}$. Since its launch on $15^{\text {th }}$ June 2006 it has been following a low-Earth quasi polar orbit, with an inclination of $70^{\circ}$, collecting data for about 10 years and measuring with unprecedented precision and sensitivity the abundance and energy spectra of various cosmic ray species of galactic, solar and trapped origin. The Alpha Magnetic Spectrometer (AMS-02) is a state-ofthe-art particle physics detector designed to operate as an external module on the International Space Station [3]. It uses the unique environment of space to study the universe and its origin by searching for nuclear antimatter and dark matter annihilation signals while performing precision measurements of cosmic rays composition and flux. AMS-02 is continuously operating since May 19, 2011 on the upper Payload Attach Point (S3) of the ISS main truss with a collection rate of circa 16 billion cosmic ray particles per year. Such an amount of data translates into scientific results that, in a vast majority of cases, are represented by measurements of particle differential energy spectra or flux ratios, e.g. particle intensities (or their ratios) as a function of particle energy. As an example, Figure 1 shows a set of the PAMELA data as measured before 2014 (see e.g. [4]). Research data are typically made available to the scientific community through publication on scientific journals, and commonly available in the form of plots or tables. Sometimes this presentation does not achieve sufficient accessibility, openness and long-term robustness. Thus, in an effort to favour an easy discover, access and use of published cosmic ray data from PAMELA, AMS-02 and other missions, the CRDB was initially developed. Recently, it has been expanding to account for the increasing complexity of space mission data, given a new structure to ensure scalability, and evolving to provide new tools for data retrieval, visualisation and download. The CRDB, with its own characteristics and features, joins with other existing databases (see e.g. Maurin et al. and references therein [5]). The Space Science Data Center Cosmic Ray DataBase has been integrated in a multi-mission and multi-messenger environment that is represented by the SSDC Multi Mission Archive (MMIA 2.0) [6] . The MMIA 2.0 is a user-friendly interface that allows users to access the whole set of all-encompassing and diverse databases maintained at SSDC, connecting several space missions exploring the Earth atmosphere, the Solar System and the Universe throughout the entire electromagnetic spectrum and other channels, such as cosmic rays. 
This paper describes the CRDB, its structure, content and the associated on-line tools for data selection, visualization and download.

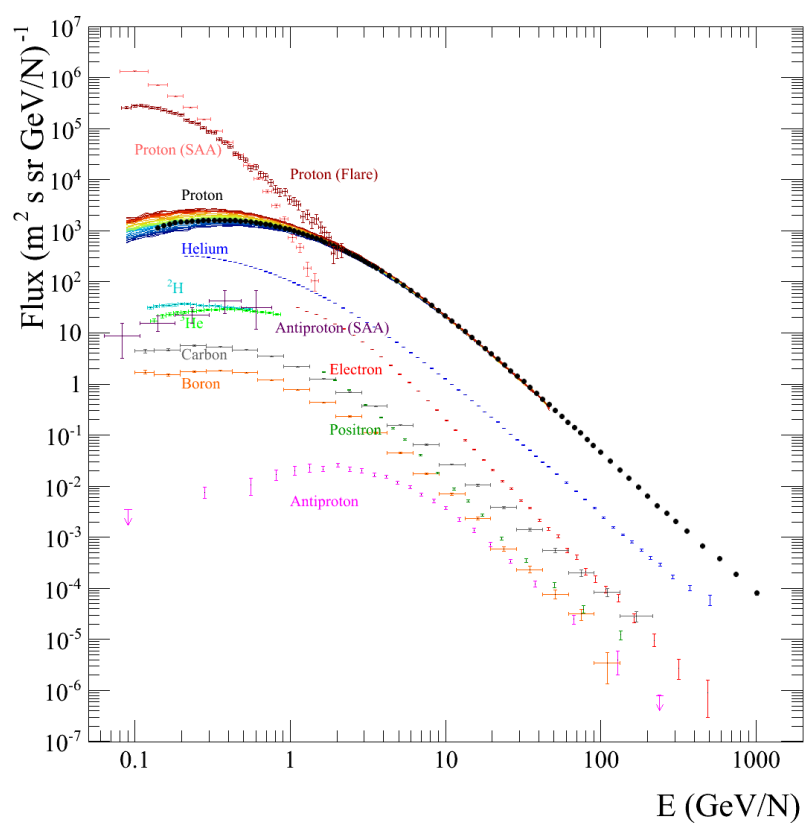

Figure 1: A set of PAMELA measurements of cosmic rays of different origin: galactic particles, solar energetic particles, particles trapped in the Earth's magnetosphere as measured in the South Atlantic Anomaly (SAA).

\section{The Cosmic Ray Database}

The following description, as a non-exhaustive introduction to the tool, has been organized being guided by the logic thread of the user web interface [1]. Cosmic ray data are accessible on-line from the CRDB home page (http://tools.asdc.asi.it/CosmicRays), upon performing data search.

\subsection{Database content and access}

The main query to the database is organized based on data typical presentation in scientific papers: the main search parameters define two quantities ' $\mathrm{X}$ ' and ' $\mathrm{Y}$ ', corresponding to the physical observables of interest, to be specified in the 'Plot' section. Other important information that can be specified at this level are the cosmic ray species ('Particle') and the mission ('Experiment') that performed the measurement. A list of the whole set of missions included in the CRDB is shown on the main page. As an example, Fig. 2 shows a snapshot of the user interface, where electron $\left(e^{-}\right)$fluxes as a function of particle kinetic energy have been selected. The main search parameters are dynamically related among each other: once some have been selected, the interface only shows the remaining allowed options for the other parameters. Queries to the database are automatically performed once a minimum set of parameters has been indicated ('Particle' and 'Plot'). 
Data and their connections are organized and stored in a relational database, exploiting the MySQL server hosted at the Space Science Data Center. Input data to the CRDB are provided as $x m l$ files, containing the measurement information as reported in the original publication tables, or as obtained by private communication with the authors. The $x m l$ file format allows for flexibility and scalability, accounting for possible different features and measured quantities, even in the case of similar scientific results. The result of the query is presented in a table. For the selected plot, particle and mission, each row represents a 'Data set': a set of ' $X$ ' and ' $Y$ ' measured values associated to their statistical and systematical errors. ' $\mathrm{Y}$ ' values can be, at the moment, differential fluxes, with default measurement units $\left(m^{2} s s r G e V\right)^{-1}$, or adimensional flux ratios. Possible values for the ' $\mathrm{X}$ ' physical quantity are kinetic energy $(\mathrm{E})$, rigidity $(R=p c / Z e$, where $p$ is the momentum, $c$ the speed of light, $Z e$ the electrical charge), and time. Default units are $\mathrm{GeV}$ and $\mathrm{GeV} / \mathrm{n}$ for kinetic energy, GV and GV/n for rigidity. It has to be noticed that there is no measurement uncertainty associated to these values, instead, interval widths are provided. The various tables of the relational database also contain additional information, where available in the original publication e.g. orbital information, or temporal interval for the measurement. Such additional information can be used, depending on the data set, to perform a 'Refined Search', limiting the output table to a sub-set of the original. Reference to the original publication is also always provided.

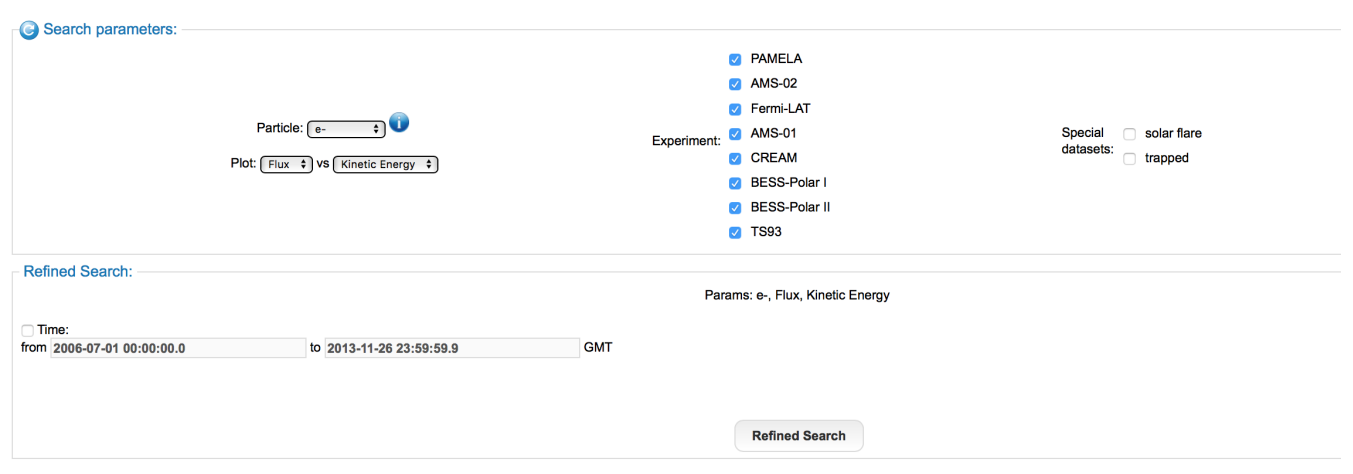

Figure 2: A screenshot of the CRDB on-line tool showing an example of data search for cosmic ray electron $\left(e^{-}\right)$fluxes as a function of kinetic energy, performed on the whole set of available experiments.

\subsection{Data visualization}

Once the query has been performed, interesting data can be selected and visualized, as shown in Fig. 3. Selected data are visualized as graphs in a plot. Thanks to an option panel it is also possible to perform some simple operation and modify the visualization. Horizontal point is the arithmetical center of the bin when no other bin center is indicated. Vertical error bars are by default total errors: i.e. when systematical errors are available, the error bar is the quadratic sum of statistical and systematical errors. If systematical errors are not available, only statistical are shown.

\subsection{Output files}

A panel allows the user to select among different format for the output files. All data are downloaded in a single zip file. Available format for the download are: 


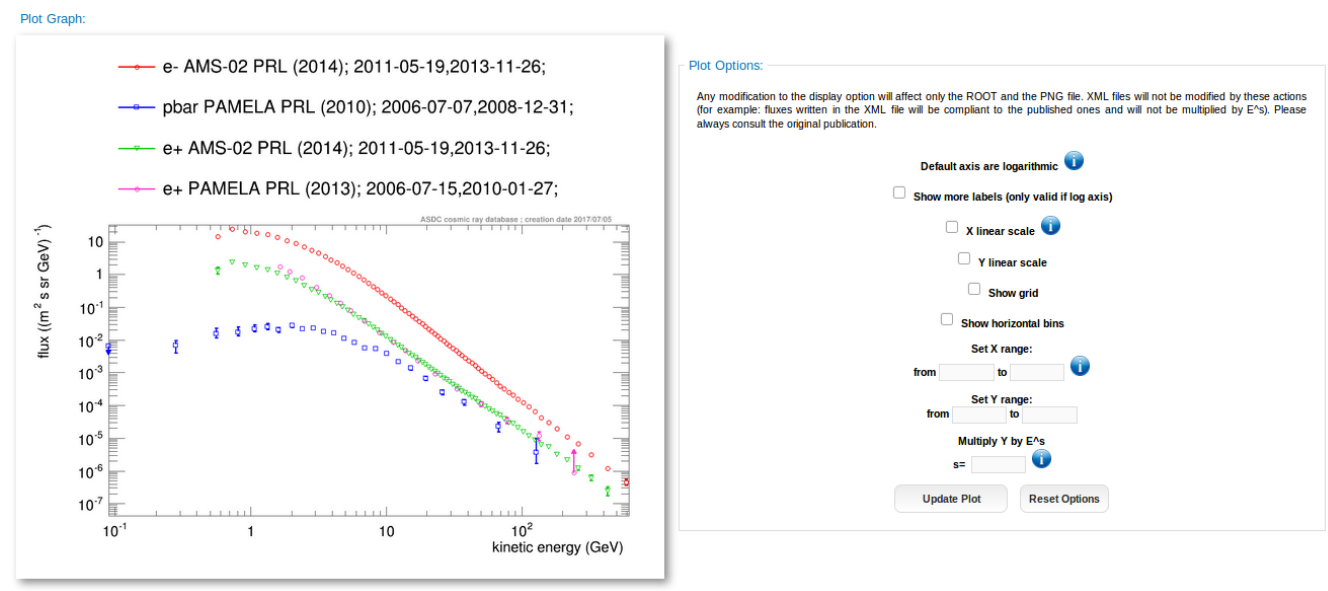

Figure 3: A screenshot of the CRDB data visualization section. Left panel: a graph corresponding to data selected by the user. Right panel: option panel to modify the graph.

png One png image of the plot displayed in the web interface.

ROOT A file in ROOT format [7] containing a TGraphAsymErrors for each selected subset, a TMultiGraph that includes all the graphs and a TCanvas as displayed in the web interface.

xml One $x m l$ file for each data subset. The $x m l$ file is organized in blocks: a <HEADER $>$ with general descripion of the dataset (Particle, Mission and Publication reference) and one or more $<$ DATA $>$ blocks, each corresponding to a data point. Used xml tags are listed in a description file available in the zip file. The $\mathrm{xml}$ file contains all available data for the subset and statistical and systematical errors are always indicated separately.

txt One text file for each graph with the transcription of the graph content. The file contains a transcription of the data points (x, y, x error, y error) as plotted in the png. Errors are total errors (statistical and systematical summed in quadrature when both available) or only statistical, depending on what required by the user in the Plot Panel Options.

In the downloaded zip file, a description file is made available when txt or xml formats are required. This file contains a list all the downloaded datasets, i.e. for each required dataset is indicated the $\mathrm{xml}$ (txt) file name and a short content description such as 'Particle', 'Mission' and 'Reference'.

It is worth stressing that, while $x m l$ files retrieve the original published information, the other output files might not, depending on the actions of the users on the graphs.

\section{Conclusion and prospects}

The CRDB at SSDC is a tool for preserving and accessing research data. It aims at offering a simple and intuitive interface. It is now growing to include more and more experiments on charged cosmic rays with the ambition to offer an exaustive archive of all published data. We plan to improve and add more graphic options and in the near future to allow for interactive grafic tools. A larger selection of output data format is foreseen. User feedback is very important to plan improvements and priorities, we welcome any comment to cosmic-rays-db@asdc.asi.it. 


\section{References}

[1] https://tools.asdc.asi.it/CosmicRays/

[2] P. Picozza and others 2007 Astropart. Phys. 27296

[3] M. Aguilar et al 2013 Phys. Rev. Lett. 110141102

[4] O. Adriani et al 2014 Phys. Rep. 5444

[5] D. Maurin et al 2014 A\&A 569 A32

[6] http://www.asdc.asi.it/mma.html

[7] R. Brun and F. Rademakers 1997 Nucl. Inst. Meth. in Phys. Res. A 389 81-86. See also http://root.cern.ch/ 